\title{
Quantitative analysis of cell-free DNA in the plasma of gastric cancer patients
}

\author{
JONG-LYUL PARK ${ }^{1,2^{*}}$, HYUN JA KIM ${ }^{3 *}$, BO YOUL CHOI ${ }^{3}$, HAN-CHUL LEE $^{1}$, HAY-RAN JANG ${ }^{1}$, \\ KYU SANG SONG ${ }^{4}$, SEUNG-MOO NOH ${ }^{5}$, SEON-YOUNG KIM ${ }^{1,2}$, DONG SOO HAN ${ }^{6}$ and YONG SUNG KIM ${ }^{1,2}$ \\ ${ }^{1}$ Medical Genomics Research Center, Korea Research Institute of Bioscience and Biotechnology (KRIBB); \\ ${ }^{2}$ Department of Functional Genomics, University of Science and Technology (UST), Daejeon 305-806; \\ ${ }^{3}$ Department of Preventive Medicine, Hanyang University College of Medicine, Seoul 133-791; \\ Departments of ${ }^{4}$ Pathology, and ${ }^{5}$ General Surgery, Chungnam National University, Daejeon 301-747; \\ ${ }^{6}$ Department of Internal Medicine, Hanyang University Guri Hospital, Gyeonggi-do 471-701, Republic of Korea
}

Received December 12, 2011; Accepted January 30, 2012

DOI: 10.3892/ol.2012.592

\begin{abstract}
In the present study, an accurate and reproducible method for quantifying cell-free DNA (cfDNA) in human blood was established and tested for its ability to predict gastric cancer in patients. Using 'Alu81-qPCR' to amplify 81-bp Alu DNA sequences, we first estimated the amount of cfDNA in the serum or plasma of 130 patients with gastric cancer to identify which source of cfDNA is more suitable for the biomarker screening of these patients. The results of Alu81-qPCR revealed that the amount of cfDNA in the plasma was low compared with that in the serum, but was found at similar levels among the samples, indicating that the plasma may be a more suitable source of cfDNA for biomarker screening. For the 54 patients with gastric cancer and the 59 age-matched healthy controls, the mean levels of plasma cfDNA were 2.4-fold higher in the patient group compared with the control group, indicating that plasma cfDNA levels may be useful for predicting patients with gastric cancer. The results of our study suggest that Alu81qPCR is a more reliable method than other techniques, such as the PicoGreen assay, for quantifying cfDNA in human blood, demonstrating the potential to complement current diagnostic procedures for the management of gastric cancer patients.
\end{abstract}

Correspondence to: Professor Yong Sung Kim, Medical Genomics Research Center, Korea Research Institute of Bioscience and Biotechnology (KRIBB), 125 Gwahak-ro, Yuseong-gu, Daejeon 305-806, Republic of Korea

E-mail: yongsung@kribb.re.kr

*Contributed equally

Key words: gastric cancer, cell-free DNA, serum, plasma, Alu sequence

\section{Introduction}

Advances in diagnostic and treatment technologies for gastric cancer have resulted in excellent long-term patient survival, but gastric cancer remains the second most common cause of cancer-related mortality worldwide (1). One of the major factors limiting the survival of gastric cancer patients may be late tumor detection. Instrumental methods are unable to detect cancer in situ; however, as cancer-related molecules, including cell-free DNA (cfDNA), from a tumor may enter the blood circulating through the affected organs, it is possible that these molecules may be detected in samples of systemic blood.

Since the first observation that the cfDNA concentration was higher in the serum of cancer patients than in that of healthy individuals (2), the cfDNA concentration has been investigated in patients with various types of cancer (3-6). cfDNA is often used as a DNA source to detect cancer cellderived mutations $(7)$, promoter methylation $(8,9)$ and loss of heterozygosity (10). Increased cfDNA levels are observed not only in patients with tumors but also following exhaustive exercise, in elderly patients suffering from acute or chronic illnesses and in individuals with premalignant lesions, inflammation or trauma (11-13). Thus, cfDNA concentration is a promising marker for the diagnosis and prognosis of cancer.

However, the reported absolute amounts of cfDNA vary among studies, probably due to the various types of cancer examined and the variety of techniques employed. cfDNA in serum and plasma has been quantified using various techniques, including spectrophotometry $(14,15)$, the PicoGreen assay $(6,16)$ and quantitative PCR (qPCR) (17). In particular, qPCR has been used to target various sequences, including $\beta$-globin (18,19), HLA DQ- $\alpha$ (20) and hTERT (21). Therefore, the implementation of cfDNA-based diagnosis in the clinic is a matter of debate due to the difficulty in comparing and normalizing the existing data, the lack of technical standardization and the relatively small number of samples examined in several studies. Although cfDNA levels in serum are much higher than those in plasma samples from the same patient, probably due to in vitro lysis of blood cells during the clotting 
process $(17,20,22)$, it is unclear whether cfDNA from plasma or serum more accurately reflects the clinical status of the patient.

In this study, we aimed to establish an accurate and reproducible procedure for quantifying cfDNA in human blood using Alu sequence-based qPCR.

\section{Materials and methods}

Patients with gastric cancer and healthy controls. We enrolled 130 consecutive patients with sporadic gastric cancer scheduled to undergo curative resection between 2006 and 2007 at the Chungnam National University Hospital (Daejeon, Korea). The patients comprised 99 males and 31 females, with a mean age of $58.4 \pm 12.3$ years (range, 32-83). Patients presenting with gastric cancer and healthy individuals at Hanyang University Hospital (Seoul, Korea) were also enrolled between 2003 and 2006. The healthy individuals included patients who had visited one of the clinics for orthopedic surgery, ophthalmology, dermatology, cosmetic surgery or family medicine, and who were also confirmed as having no severe stomach problems by gastroendoscopy. Among the patients and healthy individuals from the Hanyang University Hospital, 54 patients (34 males, 20 females) and 59 age-matched healthy individuals ( 39 males, 20 females) were selected for the case-control study. There was no significant difference in age between the cancer group $(61.8 \pm 12.3$ years) and the control group (61.6 \pm 12.6 years). The study protocol was approved by the Institutional Review Boards of Chungnam National University Hospital and Hanyang University Hospital. All data and specimens for the study were collected after obtaining informed consent from the participants.

Blood sample collection and isolation of serum and plasma $D N A$. For gastric cancer patients from Chungnam National University Hospital, 8-ml blood samples were drawn from a peripheral vein and divided into native (for serum) and EDTA-containing (for plasma) tubes at the time of diagnosis or prior to surgery for gastric cancer. The blood samples were subjected to two consecutive centrifugations at 3,000 rpm for $10 \mathrm{~min}$ at room temperature to remove the cellular components. Aliquots $(1 \mathrm{ml})$ of serum or plasma were stored at $-80^{\circ} \mathrm{C}$ until use. DNA was purified from $1 \mathrm{ml}$ of serum or plasma using the Qiagen Ultrasens Virus kit (Qiagen, Hilden, Germany) according to the manufacturer's instructions. For patients and healthy individuals from Hanyang University Hospital, the DNA was purified (as described above) only from the plasma samples.

Quantification of c $f D N A$. For the quantification of fragmented DNA, including cfDNA, $0.3 \mathrm{ml}$ of intact genomic DNA $(10 \mathrm{ng} / \mu \mathrm{l})($ Clontech Laboratories, Mountain View, CA, USA) was placed into $1.5-\mathrm{ml}$ microtubes in ice water and sonicated with a model 100 Ultrasonic Dismembrator (Fisher Scientific, Pittsburgh, PA, USA) at various times $(5,10,20,30$ and $50 \mathrm{sec}$ ) to generate different size distributions. The concentration of the fragmented DNA was then estimated using three methods: NanoDrop 1000 using a UV-Vis spectrophotometer (Thermo Scientific, Waltham, MA, USA), NanoDrop 3300 (Thermo Scientific) using the PicoGreen fluorophore and real-time qPCR by amplifying 81-bp Alu DNA sequences
(Alu81-qPCR). For Alu81-qPCR, we designed a primer set to produce an 81-bp amplicon of the Alu repeat sequence: forward primer, 5'-CCTGAGGTCAGGAGTTCGAG-3'; reverse primer, 5'-GCCCCGGCTAATTTTTGTAT-3'. Alu81-qPCR was performed using the CFX96 Real-Time PCR Detection system (Bio-Rad Laboratories, Hercules, CA, USA) under the following conditions: $95^{\circ} \mathrm{C}$ for $3 \mathrm{~min}, 45$ cycles of $95^{\circ} \mathrm{C}$ for $10 \mathrm{sec}, 60^{\circ} \mathrm{C}$ for $20 \mathrm{sec}$ and $72^{\circ} \mathrm{C}$ for $20 \mathrm{sec}$. The reaction was performed in $20 \mu \mathrm{l}$ containing the primer set and 2X SYBR Premix EX Taq (Takara, Shiga, Japan) according to the manufacturer's instructions.

Statistical analysis. The Mann-Whitney U test was used to examine the statistical difference between the cfDNA concentrations in the serum and plasma of patients with gastric cancer and between the gastric cancer patients and healthy individuals. $\mathrm{P}<0.05$ was considered to indicate a statistically significant result. The correlation between cfDNA concentration in the serum and plasma or between plasma cfDNA concentration and age was determined using the Pearson's correlation coefficient (R). The receiver operating characteristic (ROC) curve and the respective area under the ROC curve were calculated for plasma cfDNA to provide more accurate information to distinguish between patients with gastric cancer and the healthy controls.

\section{Results}

Alu81-qPCR procedure is superior to previous methods for quantifying cfDNA in human blood. In this study, we observed DNA ladder patterns in multiples of $\sim 200$ bp in all samples when cfDNA from the plasma or serum of patients with gastric cancer was separated on an agarose gel (Fig. 1A). This observation is in agreement with a previous study which reported that plasma or serum DNA often presents as a ladder pattern, corresponding to small multiples of lengths associated with nucleosomes, following electrophoresis (23). Thus, we first tested DNA which had been fragmented with sonication to establish the optimal conditions for quantifying the cfDNA concentration in human blood. Fig. 1B shows the DNA ladders fragmented with increasing sonication times of 5, 10, 20, 30 and $50 \mathrm{sec}$. Following $10 \mathrm{ng}$ of input DNA, the concentration in the aliquots of each fragmented DNA solution was simultaneously measured in triplicate with NanoDrop 1000 and a UV-Vis spectrophotometer, Alu81-qPCR and NanoDrop 3300 matched with the PicoGreen fluorophore.

When measured with NanoDrop 1000, no significant differences were observed in the estimates of the DNA concentrations in solutions prepared with different sonication times (Fig. 1C), indicating that NanoDrop 1000 measured disassociated free nucleotides in addition to double-stranded DNA fragments. When measured with NanoDrop 3300 and the PicoGreen fluorophore, however, a gradual decrease in DNA level was observed with increasing sonication time: $9.69 \pm 0.57 \mathrm{ng}$ (intact DNA), $8.02 \pm 0.57 \mathrm{ng}$ (sonication for $5 \mathrm{sec}$ ), $7.53 \pm 0.29 \mathrm{ng}(10 \mathrm{sec}), 7.03 \pm 0.4 \mathrm{ng}(20 \mathrm{sec})$ and $6.53 \pm 0.22 \mathrm{ng}$ $(30 \mathrm{sec})$. The concentration exhibited a marked decrease to $0.83 \pm 0.11 \mathrm{ng}$ with a DNA solution containing fragments $<500 \mathrm{bp}$ when sonicated for $50 \mathrm{sec}$ (Fig. 1C). Measurement with Alu81-qPCR revealed a gradual decrease in DNA concen- 
A

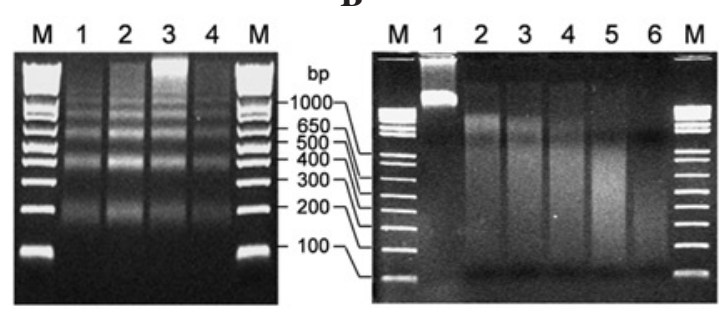

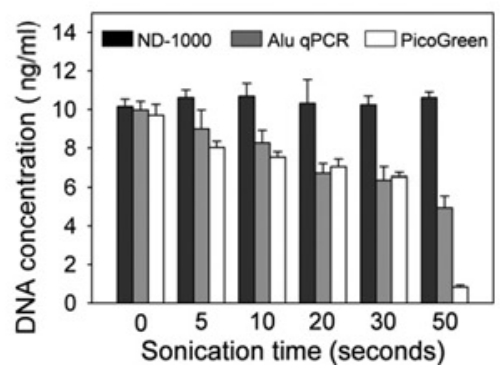

D

$\begin{array}{llllll}M & 2 & 3 & 4 & 5 & 6\end{array}$

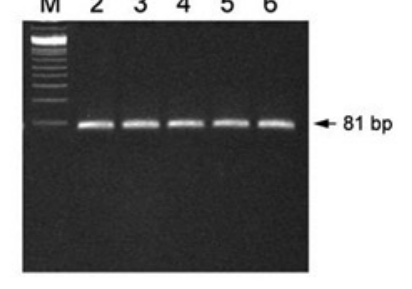

Figure 1. Quantification of fragmented DNA with Alu81-qPCR analysis. (A) Agarose gel electrophoresis of plasma or serum cfDNA from human blood. DNA (50 ng/lane) was separated on a 1\% agarose gel: lanes 1 and 2, plasma cfDNA; lanes 3 and 4, serum cfDNA; M, DNA size marker. (B) Agarose gel electrophoresis of fragmented genomic DNA. Intact genomic DNA was fragmented via sonication for various times (5, 10, 20, 30 and 50 sec). Lane 1, intact genomic DNA; lanes 2, 3, 4, 5 and 6, fragmented DNA sonicated for 5, 10, 20,30 or 50 sec, respectively. (C) Concentration of fragmented DNA as detected with three assays. Fragmented DNA was quantified in each sample with NanoDrop 1000 using a UV-Vis spectrophotometer (ND-1000), Alu81-qPCR (Alu qPCR) and NanoDrop 3300 using the PicoGreen fluorophore (PicoGreen) in triplicate. Each bar is the mean \pm SD. (D) Amplification of the 81-bp Alu fragment from the fragmented DNA. Lane numbers are the same as those of Fig. 1B. cfDNA, cell-free DNA.

A

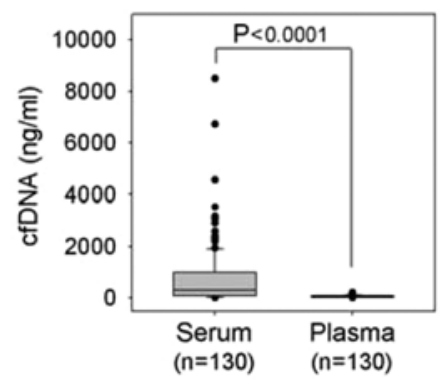

B

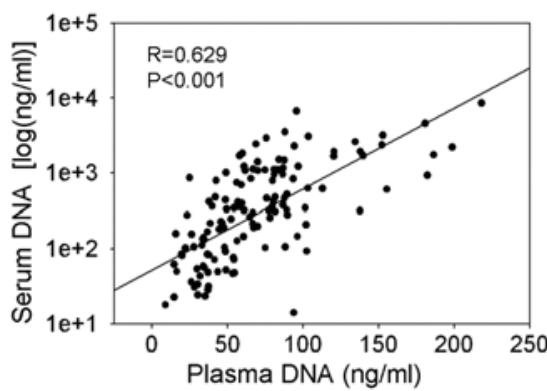

C

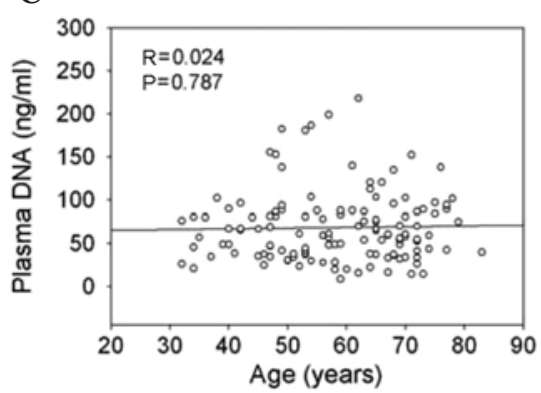

Figure 2. Comparison of cfDNA concentration in the serum and plasma of blood from patients with gastric cancer. (A) Concentration of cfDNA in serum and plasma. Serum or plasma cfDNA was simultaneously purified from the blood of patients with gastric cancer and their concentrations were measured using Alu81-qPCR. (B) Correlation between serum and plasma cfDNA concentrations. (C) Change in plasma cfDNA concentration with respect to age. Plasma cfDNA level was plotted against patient age. cfDNA, cell-free DNA.

tration with increasing sonication time that was similar to the results of the NanoDrop 3300 method, with the exception of the measurement following sonication for $50 \mathrm{sec}$, in which the concentration was estimated as $4.93 \pm 0.61 \mathrm{ng}$. This result suggests that even smaller DNA fragments may be effectively estimated with the Alu81-qPCR procedure.

We performed PCR with each sonicated DNA solution containing $10 \mathrm{ng}$ of input DNA to confirm the annealing specificity of the Alu81-qPCR primer set. Fig. 1D shows that the production of the 81-bp amplicon was highly reproducible in all DNA solutions without producing an additional noise band and that amplification was independent of sonication time. This result suggests that Alu81-qPCR is superior to the two other methods for measuring the concentration of small fragmented DNA, including cfDNA in the serum or plasma of human blood.

Plasma DNA is more suitable for measuring the concentration of $c f D N A$. To determine whether serum or plasma is more suitable for measuring cfDNA in human blood, we measured the cfDNA concentration in the serum and plasma of blood from 130 patients with gastric cancer using Alu81-qPCR. The cfDNA concentration was significantly higher in serum $(755.9 \pm 1,203.7 \mathrm{ng} / \mathrm{ml}$; range, $7.4-8,495.8)$ than in plasma $(68.7 \pm 40.7 \mathrm{ng} / \mathrm{ml}$; range, 8.9-218.1; $\mathrm{P}<0.0001$; Fig. 2A) and the log-transformed concentrations were highly correlated with each other $(\mathrm{R}=0.629, \mathrm{P}<0.0001$; Fig. $2 \mathrm{~B})$. Thus, plasma cfDNA is more suitable for measuring the concentration of cfDNA in human blood, as we observed a wider range of cfDNA concentrations in serum than in plasma. Table I shows that the cfDNA concentration tended to be higher in patients with tumor volumes $>5 \mathrm{~cm}^{2}(73.9 \pm 43.4 \mathrm{ng} / \mathrm{ml})$ than in those with tumor volumes $\leq 5 \mathrm{~cm}^{2}(61.0 \pm 34.6 \mathrm{ng} / \mathrm{ml})$, although the difference was not statistically significant. No significant change was detected in plasma DNA levels with increasing age (Fig. 2C) and no correlation was observed between cfDNA level and any other clinicopathological characteristic (Table I).

Plasma cfDNA discriminates between patients with gastric cancer and healthy individuals. To evaluate the possible role of the plasma cfDNA level as a biomarker to discriminate between patients with gastric cancer and healthy individuals, we measured the concentration of plasma cfDNA using Alu81qPCR in age- and gender-matched healthy individuals and patients with gastric cancer. The mean plasma cfDNA concentration was significantly higher in patients with gastric cancer $(71.4 \pm 55.4 \mathrm{ng} / \mathrm{ml}$; range, $16.5-220.9)$ than that in healthy individuals $(29.8 \pm 14.4 \mathrm{ng} / \mathrm{ml}$; range, 10.6-69.3; $\mathrm{P}<0.001$; Mann-Whitney U test; Fig. 3A). Of note, the mean plasma cfDNA concentration $(71.4 \mathrm{ng} / \mathrm{ml} ; \mathrm{n}=54)$ in patients from 
Table I. Quantification of plasma cfDNA in patients with gastric cancer with respect to clinicopathological characteristics.

\begin{tabular}{|c|c|c|c|}
\hline Clinicopathological parameter & Cases & Mean \pm SD of plasma cfDNA $(\mathrm{ng} / \mathrm{ml})$ & P-value ${ }^{a}$ \\
\hline Gender & & & 0.56 \\
\hline Male & 99 & $69.0 \pm 42.6$ & \\
\hline Female & 31 & $67.6 \pm 33.4$ & \\
\hline Age (years) & & & 0.74 \\
\hline$\leq 50$ & 39 & $69.8 \pm 38.2$ & \\
\hline$>50$ & 91 & $68.4 \pm 11.0$ & \\
\hline Tumor volume $\left(\mathrm{cm}^{2}\right)$ & & & 0.1 \\
\hline$\leq 5$ & 52 & $61.0 \pm 34.6$ & \\
\hline$>5$ & 78 & $73.9 \pm 43.4$ & \\
\hline Histology $y^{b}$ & & & 0.72 \\
\hline Intestinal & 61 & $66.2 \pm 37.4$ & \\
\hline Diffuse & 52 & $72.2 \pm 46.0$ & \\
\hline Tumor progression & & & 0.85 \\
\hline EGC & 60 & $67.8 \pm 36.1$ & \\
\hline AGC & 70 & $69.5 \pm 44.1$ & \\
\hline Lymph node metastasis & & & 0.59 \\
\hline Absent & 75 & $70.8 \pm 42.4$ & \\
\hline Present & 55 & $65.8 \pm 37.8$ & \\
\hline
\end{tabular}

${ }^{\mathrm{a}}$ Analyzed using the Mann-Whitney rank sum test. ${ }^{\mathrm{b}}$ Mixed type $(67.0 \pm 33.9, \mathrm{n}=17)$ was not significantly different from the intestinal or diffuse types and was therefore not included in this table. EGC, early gastric cancer; AGC, advanced gastric cancer; cfDNA, cell-free DNA.
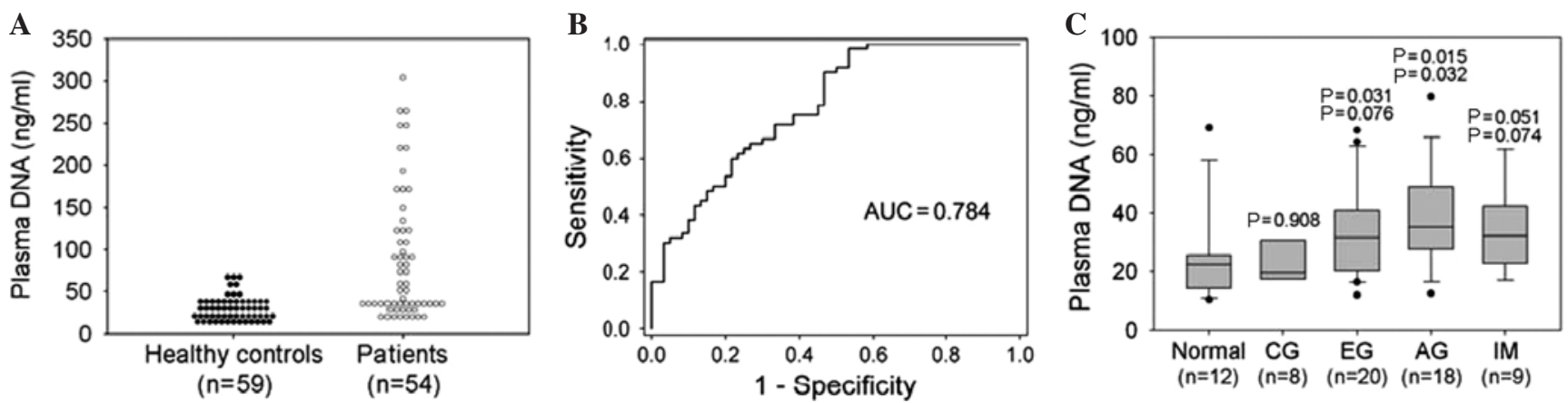

Figure 3. Concentration of plasma cfDNA in the blood of healthy individuals and patients with gastric cancer. (A) Comparison of plasma cfDNA levels in healthy individuals and patients. Concentrations of plasma DNA in patients with gastric cancer were significantly higher compared with those in healthy individuals ( $\mathrm{P}<0.001$; Mann-Whitney U test). (B) ROC curve for plasma cfDNA concentrations in healthy individuals and patients with gastric cancer. The area under the ROC curve was estimated using the logistic procedure in SigmaPlot 11.0 software. (C) Concentrations of plasma cfDNA in healthy individuals without gastric cancer. The healthy individuals in Fig. 3A were divided into five subgroups according to endoscopic and pathological observations: normal, individuals with no precancerous lesions; $\mathrm{CG}$, individuals with acute or chronic gastritis; EG, individuals with erosive gastritis; AG, individuals with atrophic gastritis; IM, individuals with intestinal metaplasia. As an individual may have more than one type of precancerous lesions simultaneously, certain individuals were counted more than once in the subgroups. The P-value above the CG box indicates the result from the Mann-Whitney U test vs. the normal group. P-values above the EG, AG and IM boxes show results vs. the normal (top P-value) and CG groups (bottom P-value). The box plot analysis shows the median and the outliers. cfDNA, cell-free DNA; ROC, receiver operating characteristic; AUC, area under the ROC curve.

Hanyang University Hospital was not significantly different from that $(68.7 \mathrm{ng} / \mathrm{ml} ; \mathrm{n}=130)$ in patients from Chungnam National University Hospital (Mann-Whitney U test, $\mathrm{P}=0.367$ ).

In this study, the optimal cut-off value for discriminating between patients and healthy individuals was a cfDNA concentration of $32.3 \mathrm{ng} / \mathrm{ml}$. The sensitivity of the test was estimated as $75 \%$ (41/54 patients with gastric cancer with plasma DNA values higher than the cut-off) and the specificity as $63 \%$ (37/59 healthy individuals with plasma DNA values lower than the cut-off). The area under the ROC curve was 0.784 (Fig. 3B).

Quantification of plasma cfDNA in healthy individuals. Although healthy individuals were considered to have no neoplasms as observed with stomach endoscopy, they often had various types of precancerous lesions, including chronic $(\mathrm{CG})$, erosive $(\mathrm{EG})$ or atrophic $(\mathrm{AG})$ gastritis or intestinal metaplasia (IM). In this study, only $20 \%(12 / 59)$ of the healthy 
individuals had no precancerous lesions and were thus defined as normal. The mean plasma cfDNA concentration in this normal group was $24.2 \pm 15.5 \mathrm{ng} / \mathrm{ml}$ (Fig. 3C), which was not significantly different from that $(23.5 \pm 9.4 \mathrm{ng} / \mathrm{ml})$ in the $\mathrm{CG}$ group. However, the plasma cfDNA concentrations for the EG, AG and IM groups were significantly higher than those for the normal group $(E G, 34.1 \pm 15.1 \mathrm{ng} / \mathrm{ml}, \mathrm{P}=0.031 ; \mathrm{AG}$, $38.8 \pm 17.9 \mathrm{ng} / \mathrm{ml}, \mathrm{P}=0.015 ; \mathrm{IM}, 34.7 \pm 13.9 \mathrm{ng} / \mathrm{ml}, \mathrm{P}=0.051$; Fig. 3C).

\section{Discussion}

Alu81-qPCR is a highly sensitive and reproducible method for quantifying cfDNA in human blood. The Alu sequence is the most abundant repeated sequence in the human genome, with approximately 1.4 million copies per genome (24). We observed that cfDNA in serum largely consisted of fragments with sizes that were multiples of approximately $200 \mathrm{bp}$, consistent with a previous study which reported that plasma and serum DNA often presents as a ladder pattern following electrophoresis, corresponding to small multiples of lengths associated with nucleosomes (23). A previous study used 115-bp Alu repeats as a target in qPCR for DNA quantification; the results revealed sufficient sensitivity for accurate quantification and high linearity with as little as $0.01 \mathrm{pg}$ of DNA (25). In the present study, Alu81-qPCR showed high sensitivity and linearity even though the DNA was severely fragmented and detected with the PicoGreen assay. This result indicates that Alu81-qPCR is superior for measuring cfDNA levels compared with the previous technologies that are based on UV-Vis spectrophotometry or the PicoGreen fluorophore.

Using Alu81-qPCR, we confirmed that the amount of cfDNA was 11-fold higher in serum $(755.9 \pm 1,203.7 \mathrm{ng} / \mathrm{ml})$ compared with plasma samples from the same patient $(68.7 \pm 40.5 \mathrm{ng} / \mathrm{ml})$ in 130 patients with gastric cancer, consistent with the results of previous studies (26-28). The cause for the significant difference between the two sources is unclear $(27,29)$. It is possible that DNA loss may have occurred during the purification from plasma but not from serum, as coagulation factors and related proteins are present in plasma. Another possible explanation is the unequal distribution of DNA during separation from whole blood. Based on these two explanations, Umetani et al (25) advocated that serum is a more suitable sample source than plasma for examining cfDNA as a biomarker.

Extraneous DNA from leukocytes or other sources can be released into serum during its separation from whole blood (28). DNA of a high molecular weight, which may be derived from leukocytes, was often observed (in addition to the 200-bp ladder) on the agarose gels of serum DNA. The wide range $(7.4-8,495.8 \mathrm{ng} / \mathrm{ml})$ of DNA concentrations in serum may also be due to the high molecular weight DNA. However, we cannot exclude the possibility of extraneous DNA contamination in plasma during its separation from whole blood since the range of DNA concentrations in plasma was also wide (8.9-218.1 $\mathrm{ng} / \mathrm{ml})$, although the upper limit was lower than that in serum. Moreover, our results show that the log-transformed concentration of DNA in serum was highly correlated with that of the plasma DNA from the same patient, as reported previously (25), suggesting that the presence of extraneous DNA in plasma and serum may be due to another preanalytical factor, for example the delayed separation of plasma or serum from whole blood or the freeze-thaw procedure (29). It is possible that extraneous DNA may be released into the serum or plasma during the separation from whole blood, but the amount released into serum is likely to be greater than that released into plasma. Thus, we suggest that plasma is a more suitable source for cfDNA for use as a biomarker, as serum and plasma DNA are not equivalent in terms of their diagnostic potential.

We also observed that the plasma cfDNA level was higher in patients with gastric cancer than in healthy controls, consistent with the results of previous studies (3-6,30,31). In our study, we found that the mean concentration of plasma cfDNA was 2.4-fold higher in patients with gastric cancer $(71.4 \pm 55.4 \mathrm{ng} / \mathrm{ml})$ than that in healthy individuals $(29.8 \pm 14.4 \mathrm{ng} / \mathrm{ml})$. Based on the cut-off value of $32.3 \mathrm{ng} / \mathrm{ml}$ for plasma DNA, we suggest that patients with gastric cancer may be discriminated from healthy individuals with $75 \%$ sensitivity and $63 \%$ specificity using the Alu81-qPCR test. In addition, our data showed significantly increased levels of plasma cfDNA in groups with precancerous lesions, including the EG, AG and IM groups, compared with the group with normal mucosa, in agreement with previous results (11-13).

cfDNA is not currently used in clinical practice due to various critical methodological and technical aspects that are related to the evaluation of the quantity in the bloodstream. The various analytical and preanalytical methods used by different groups have not permitted a direct comparison of results. No study has evaluated the reproducibility of the data obtained by another group using the same method on different samples with a different instrument. Our findings have shown that the cfDNA levels in serum fluctuated more than those in the plasma of gastric cancer patients, indicating that cfDNA levels in the serum and plasma are not equivalent in terms of their diagnostic potential, although the concentrations in the two types of samples were significantly correlated with each other. Thus, our data suggest that the quantification of plasma cfDNA can be standardized and that Alu81-qPCR has the potential to complement current diagnostic procedures for the management of gastric cancer patients.

\section{Acknowledgements}

This study was supported by the Future-based Technology Development Program of the National Research Foundation of Korea (NRF2011-0015710) that is funded by the Ministry of Education, Science and Technology, as well as a KRIBB Research Initiative Grant.

\section{References}

1. Parkin DM, Bray F, Ferlay J and Pisani P: Global cancer statistics, 2002. CA Cancer J Clin 55: 74-108, 2005.

2. Leon SA, Shapiro B, Sklaroff DM and Yaros MJ: Free DNA in the serum of cancer patients and the effect of therapy. Cancer Res 37: 646-650, 1977

3. Fournié GJ, Courtin JP, Laval F, et al: Plasma DNA as a marker of cancerous cell death. Investigations in patients suffering from lung cancer and in nude mice bearing human tumours. Cancer Lett 91: 221-227, 1995.

4. Jung K, Stephan C, Lewandowski M, et al: Increased cell-free DNA in plasma of patients with metastatic spread in prostate cancer. Cancer Lett 205: 173-180, 2004. 
5. Laktionov PP, Tamkovich SN, Rykova EY, et al: Extracellular circulating nucleic acids in human plasma in health and disease Nucleosides Nucleotides Nucleic Acids 23: 879-883, 2004.

6. Xie GS, Hou AR, Li LY, Gao YN and Cheng SJ: Quantification of plasma DNA as a screening tool for lung cancer. Chin Med J (Engl) 117: 1485-1488, 2004.

7. Diehl F, Schmidt K, Choti MA, et al: Circulating mutant DNA to assess tumor dynamics. Nat Med 14: 985-990, 2008.

8. Lofton-Day C, Model F, Devos T, et al: DNA methylation biomarkers for blood-based colorectal cancer screening. Clin Chem 54: 414-423, 2008.

9. Chan KC, Lai PB, Mok TS, et al: Quantitative analysis of circulating methylated DNA as a biomarker for hepatocellular carcinoma. Clin Chem 54: 1528-1536, 2008.

10. Schwarzenbach H, Chun FK, Müller I, et al: Microsatellite analysis of allelic imbalance in tumour and blood from patients with prostate cancer. BJU Int 102: 253-258, 2008.

11. Atamaniuk J, Vidotto C, Tschan H, Bachl N, Stuhlmeier KM and Müller MM: Increased concentrations of cell-free plasma DNA after exhaustive exercise. Clin Chem 50: 1668-1670, 2004.

12. Jiang $\mathrm{N}$ and Pisetsky DS: The effect of inflammation on the generation of plasma DNA from dead and dying cells in the peritoneum. J Leukoc Biol 77: 296-302, 2005.

13. Marx J: Cancer research. Inflammation and cancer: the link grows stronger. Science 306: 966-968, 2004

14. Silva JM, Dominguez G, Garcia JM, et al: Presence of tumor DNA in plasma of breast cancer patients: clinicopathological correlations. Cancer Res 59: 3251-3256, 1999.

15. Silva JM, Silva J, Sanchez A, et al: Tumor DNA in plasma at diagnosis of breast cancer patients is a valuable predictor of disease-free survival. Clin Cancer Res 8: 3761-3766, 2002.

16. Chang HW, Lee SM, Goodman SN, et al: Assessment of plasma DNA levels, allelic imbalance, and CA 125 as diagnostic tests for cancer. J Natl Cancer Inst 94: 1697-1703, 2002.

17. Thijssen MA, Swinkels DW, Ruers TJ and de Kok JB: Difference between free circulating plasma and serum DNA in patients with colorectal liver metastases. Anticancer Res 22: 421-425, 2002.

18. Allen D, Butt A, Cahill D, Wheeler M, Popert R and Swaminathan R: Role of cell-free plasma DNA as a diagnostic marker for prostate cancer. Ann N Y Acad Sci 1022: 76-80, 2004

19. Gal S, Fidler C, Lo YM, et al: Quantitation of circulating DNA in the serum of breast cancer patients by real-time PCR. Br J Cancer 90: 1211-1215, 2004.
20. Lee TH, Montalvo L, Chrebtow V and Busch MP: Quantitation of genomic DNA in plasma and serum samples: higher concentrations of genomic DNA found in serum than in plasma. Transfusion 41: 276-282, 2001.

21. Sozzi G, Conte D, Leon M, et al: Quantification of free circulating DNA as a diagnostic marker in lung cancer. J Clin Oncol 21: 3902-3908, 2003

22. Lui YY, Chik KW, Chiu RW, Ho CY, Lam CW and Lo YM: Predominant hematopoietic origin of cell-free DNA in plasma and serum after sex-mismatched bone marrow transplantation. Clin Chem 48: 421-427, 2002.

23. Giacona MB, Ruben GC, Iczkowski KA, Roos TB, Porter DM and Sorenson GD: Cell-free DNA in human blood plasma: length measurements in patients with pancreatic cancer and healthy controls. Pancreas 17: 89-97, 1998.

24. Gu Z, Wang H, Nekrutenko A and Li WH: Densities, length proportions, and other distributional features of repetitive sequences in the human genome estimated from 430 megabases of genomic sequence. Gene 259: 81-88, 2000.

25. Umetani N, Hiramatsu S and Hoon DS: Higher amount of free circulating DNA in serum than in plasma is not mainly caused by contaminated extraneous DNA during separation. Ann N Y Acad Sci 1075: 299-307, 2006.

26. Taback B, O'Day SJ and Hoon DS: Quantification of circulating DNA in the plasma and serum of cancer patients. Ann N Y Acad Sci 1022: 17-24, 2004

27. Gautschi O, Bigosch C, Huegli B, et al: Circulating deoxyribonucleic acid as prognostic marker in non-small-cell lung cancer patients undergoing chemotherapy. J Clin Oncol 22: 4157-4164, 2004.

28. Holdenrieder S, Stieber P, Chan LY, et al: Cell-free DNA in serum and plasma: comparison of ELISA and quantitative PCR. Clin Chem 51: 1544-1546, 2005.

29. Chan KC, Yeung SW, Lui WB, Rainer TH and Lo YM: Effects of preanalytical factors on the molecular size of cell-free DNA in blood. Clin Chem 51: 781-784, 2005.

30. Pathak AK, Bhutani M, Kumar S, Mohan A and Guleria R: Circulating cell-free DNA in plasma/serum of lung cancer patients as a potential screening and prognostic tool. Clin Chem 52: $1833-1842,2006$.

31. Gormally E, Caboux E, Vineis P and Hainaut P: Circulating free DNA in plasma or serum as biomarker of carcinogenesis: practical aspects and biological significance. Mutat Res 635: 105-117, 2007. 